\title{
THEORETICAL AND EXPERIMENTAL STUDY OF MULTIMODAL TARGETED ENERGY TRANSFER IN A SYSTEM OF COUPLED OSCILLATORS
}

\author{
G. Kerschen ( $\left.{ }^{1}\right)$, J.J. Kowtko $\left({ }^{2}\right)$, D.M. McFarland $\left({ }^{2}\right)$, L.A. Bergman $\left({ }^{2}\right)$, A.F. Vakakis $\left({ }^{2,3}\right)$ \\ ( $\left.{ }^{1}\right)$ Department of Materials, Mechanical and Aerospace Engineering, University of \\ Liege, Belgium; E-mail: g.kerschen@ulg.ac.be \\ (2) Department of Aerospace Engineering, University of Illinois at Urbana- \\ Champaign, U.S.A.; E-mail: kowtko, dmmcf, lbergman, avakakis@uiuc.edu \\ $\left(^{3}\right)$ Division of Mechanics, National Technical University of Athens, Greece; E- \\ mail: vakakis@central.ntua.gr
}

\section{EXTENDED ABSTRACT}

The tuned mass damper (TMD), which arose from the seminal works of Frahm and Den Hartog, is a simple and efficient device, but it is only effective when it is precisely tuned to the frequency of a vibration mode. Another limitation of the TMD occurs when several modes of a multi-degree-of-freedom (MDOF) structure participate in the system response. In this case, the TMD is incapable of absorbing the vibrations of more than one mode of the primary system, a feature which clearly limits its efficiency.

The idea of a nonlinear vibration absorber dates back to Roberson in 1952 and was examined in several studies since then. Recently, it was shown that targeted energy transfer from a primary structure to a nonlinear attachment can be realized by using essential nonlinearity, characterized by the absence of a linear term in the forcedisplacement relation [1]. This nonlinear vibration absorber, termed a nonlinear energy sink (NES), is markedly different from those previously proposed in the literature.

When an NES is connected to a linear oscillator, the underlying Hamiltonian system can exhibit complicated dynamics, including a countable infinity of periodic orbits and strong motion localization in either oscillator [2]. In the presence of viscous dissipation, the richness of the dynamics can make the NES interact with and extract energy from the primary structure over a relatively wide frequency range; this represents a distinct improvement over the TMD and increases the robustness of vibration absorption in the presence of transient, broadband disturbances. The underlying dynamical mechanisms were shown to be transient resonance captures and nonlinear beat phenomena, which lead to irreversible and reversible energy transfers, respectively [3].

The improvement in robustness brought about by an NES is not limited to single-degree-of-freedom (SDOF) structures. The lack of any preferential resonance frequency of the NES makes it capable of resonating with any mode of the primary structure through isolated resonance captures. Moreover, in the case of multimodal response, resonance capture cascades during which the nonlinear attachment resonates with different modes sequentially (i.e., it extracts energy from a mode before proceeding to the next mode) were also observed in these studies. This represents further evidence of the versatility of the NES.

The purpose of this study is the theoretical and experimental investigation of targeted energy transfers from a two-degree-of-freedom (2DOF) primary structure to an NES. It is demonstrated that an NES can resonate with and extract energy from both modes of the primary structure. By facilitating these energy transfers, notably through excitation of appropriate periodic and quasi-periodic impulsive orbits, one can promote dissipation of a major portion of externally induced energy in the nonlinear attachment.

The system considered herein is composed of a 2DOF linear system coupled to an NES,

$$
\begin{aligned}
& m_{1} \ddot{y}_{1}+\varepsilon \lambda_{1} \dot{y}_{1}+k_{1} y_{1}+k_{12}\left(y_{1}-y_{2}\right)=0 \\
& m_{2} \ddot{y}_{2}+\varepsilon \lambda_{2} \dot{y}_{2}+\varepsilon \lambda\left(\dot{y}_{2}-\dot{v}\right)+k_{2} y_{2}+k_{12}\left(y_{2}-y_{1}\right)+C\left(y_{2}-v\right)^{3}=0 \\
& \varepsilon \ddot{v}+\varepsilon \lambda\left(\dot{v}-\dot{y}_{2}\right)+C\left(v-y_{2}\right)^{3}=0
\end{aligned}
$$

Variables $y_{1}$ and $y_{2}$ refer to the displacement of the primary system, whereas $v$ refers to the NES displacement. For obvious practical reasons, a light-weight NES is considered by requiring that $\varepsilon<<1$.

An important finding drawn from previous studies of a SDOF oscillator coupled to an NES [2,3] is that a precise picture of the vigorous energy transfers that may take place in the system can be obtained by gathering in a frequency-energy plot the nonlinear normal modes (NNMs) of the underlying Hamiltonian system together with the locus of impulsive orbits, defined as accommodating impulsive initial conditions on the primary system. Such a frequency-energy plot is given in Figure 1 for $m_{1}=m_{2}=k_{1}=k_{2}=k_{12}=C=1, \varepsilon=0.05$ from which it can be concluded that (i) targeted energy transfer can be realized for in-phase and anti-phase motions of the primary system, which shows the adaptability of the NES; (ii) two families of impulsive orbits, namely in-phase and antiphase impulsive orbits, exist.-Figure 2 depicts the experimental fixture that was built to validate these findings. The 
NES response represented in a frequency-energy plane using the wavelet transform demonstrates that the NES can indeed interact sequentially with both modes of the primary system.

\section{REFERENCES}

[1] Vakakis, A.F., Gendelman, O., 2001, 'Energy Pumping in Nonlinear Mechanical Oscillators: Part II Resonance Capture,' Journal of Applied Mechanics 68, 42-48.

[2] Lee, Y.S., Kerschen, G., Vakakis, A.F., Panagopoulos, P., Bergman, L.A., McFarland, D.M., 2005, 'Complicated Dynamics of a Linear Oscillator with an Essentially Nonlinear Local Attachment,' Physica D 204, 41-69.

[3] Kerschen, G., Lee, Y.S., Vakakis, A.F., McFarland, D.M., Bergman, L.A., 2005, 'Irreversible Passive Energy Transfer in Coupled Oscillators with Essential Nonlinearity,' SIAM Journal on Applied Mathematics, in press.

\section{FIGURES}

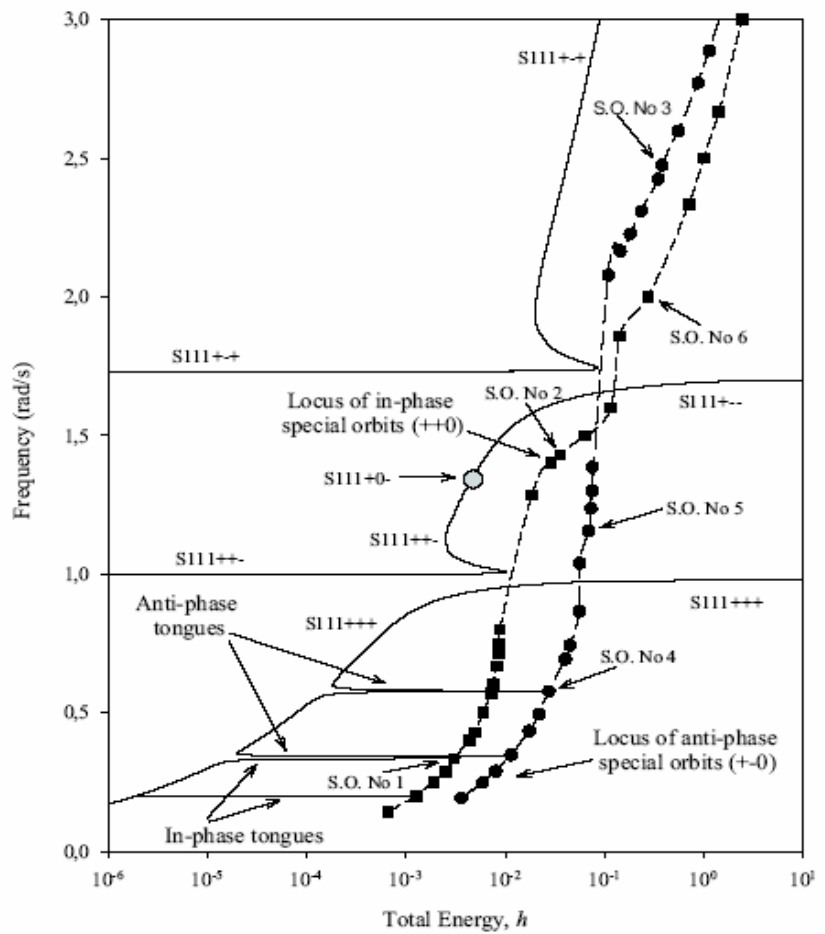

Figure 1: Frequency-energy plot: NNMs on the backbone branch and the manifold of impulsive orbits
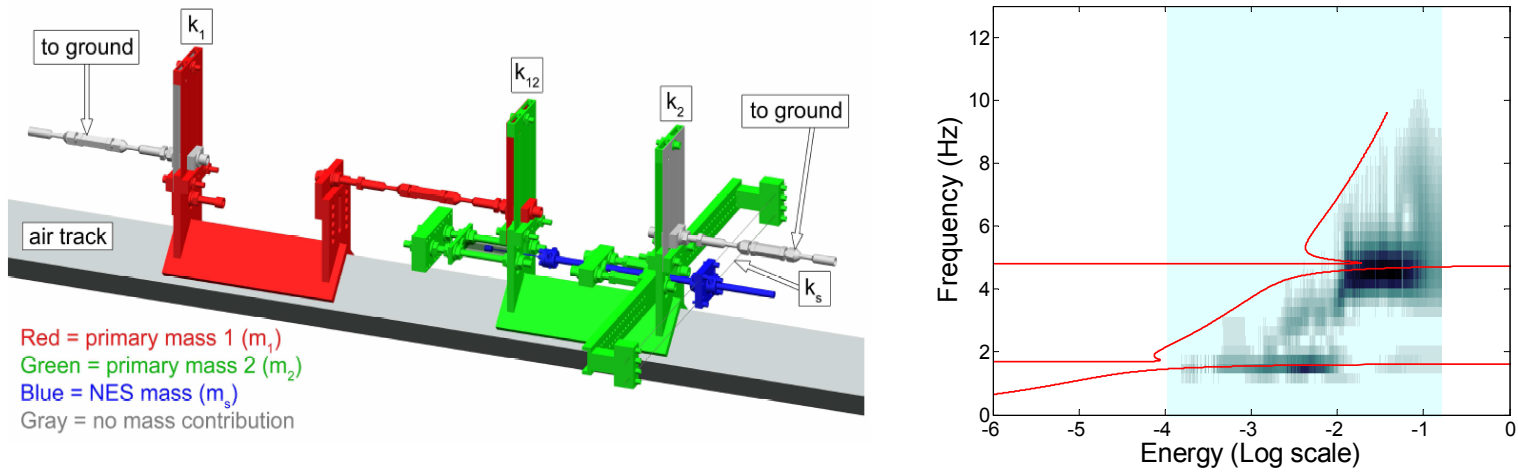

Figure 2: Experimental fixture; experimental NES response represented in a frequency-energy plane using the wavelet transform. The solid lines depict the backbone of the system (i.e., the periodic solutions of the underlying Hamiltonian system which correspond to synchronous motion of both oscillators) 